\title{
Direct evidence to support the restriction of intramolecular rotation hypothesis for the mechanism of aggregation-induced emission: temperature resolved terahertz spectra of tetraphenylethene
}

\author{
Edward P. J. Parrott, ${ }^{a}$ Nicholas Y. Tan, ${ }^{b}$ Rong Rong Hu, ${ }^{c}$ J. Axel Zeitler, ${ }^{* b}$ Ben Zhong Tang $*^{c}$ and Emma \\ Pickwell-MacPherson* ${ }^{a}$
}

Received (in $X X X, X X X)$ Xth $X X X X X X X X X 20 X X$, Accepted Xth XXXXXXXXX 20XX

DOI: $10.1039 / b 000000 x$

In contrast to the traditional fluorescent dyes that exhibit a decrease in fluorescence upon aggregation, Aggregation10 Induced Emission (AIE) molecules are a family of fluorophors which exhibit increased fluorescence upon aggregation. Consequently, AIE molecules represent an interesting new material with potential applications in fluorescent chemo/biosensors, light emitting devices and 15 medical diagnostics. Numerous mechanisms have been proposed to explain this phenomenon, including isomerisation, and restriction of intramolecular rotations (RIR). However, there has not been any direct experimental evidence to support either one of these hypotheses. Here we 20 use terahertz time-domain-spectroscopy (THz-TDS) and solid-state computational simulations of an AIE molecule to link the increase in intensity of intramolecular rotation and rocking modes to the measured fluorescence and reveal direct evidence supporting the RIR hypothesis. This is the first time

25 that terahertz spectroscopy has been used to directly probe such molecular motions in AIE materials and in doing so we have found conclusive evidence to fully explain the AIE mechanism.

\section{Introduction}

${ }_{30}$ Organic luminescent materials play a major role in applications ranging from fluorescent sensors ${ }^{1,2}$ to imaging applications ${ }^{3,4}$, due to advantages such as low cost, ease of fabrication, flexibility, high sensitivity and selectivity. These materials are normally used in either the solid state or in aqueous media, however efficient 35 emission from these aggregated states is often difficult as many compounds quench their own fluorescence in the condensed phase due to non-radiative decay pathways ${ }^{5-7}$. Many chemical, physical and engineering solutions have been proposed to alleviate this issue, but these have met with only limited success ${ }^{8-}$ ${ }_{40}^{10}$.

In 2001, a novel system was reported by one of the present authors in which the aggregation of the luminescent molecule enhanced the intensity of the emitted light ${ }^{11}$. These molecules, including tetraphenylethene (TPE) and hexaphenyl silole (HPS), 45 are non-emissive when dissolved in good solvents but become highly emissive when aggregated in poor solvents or in the solid state. This phenomenon was called aggregation-induced emission (AIE) ${ }^{12}$. Since this discovery, several mechanisms have been proposed by different research groups, including restriction 50 of intramolecular rotations (RIR), conformational planarization, $J$-aggregate formation, hydrophobic effects, $E / Z$ isomerisation and twisted intramolecular charge transfer ${ }^{13-16}$. Despite some indirect experimental and computational evidence in support of the RIR hypothesis, there lacks direct evidence to support or 55 refute the proposed mechanisms ${ }^{12,15,17-20}$. It is imperative that detailed mechanistic studies of the AIE phenomenon are undertaken to benefit and direct further exploration of high-tech applications, such as using AIE compounds in chemical ${ }^{21}$, chirality ${ }^{22}$ or gamma ray ${ }^{23}$ sensing applications.

60 Terahertz time-domain spectroscopy (THz-TDS) has been used to study the material, chemical and electrical properties of a variety of sample systems, including crystalline polymorphs ${ }^{24-27}$, glasses $^{28}$, carbon nanotubes ${ }^{29-32}$ and proteins ${ }^{33,34}$. Electromagnetic radiation at a frequency of $1 \mathrm{THz}\left(10^{12} \mathrm{~Hz}, 4.1 \mathrm{meV}\right)$ is low 65 enough in energy to probe the electronic structure of materials, the low frequency intermolecular interactions, and also some low energy intramolecular modes. Therefore, it is sensitive to small structural changes in crystalline structures and the relaxation dynamics in condensed matter, and can measure the frequency-

70 dependent conductivity of materials in a non-contact manner. In addition, it is non-ionising due to its low photon energy when compared to other measurement techniques such as X-ray and UV-vis spectroscopy. Previous research has demonstrated that low frequency rotational modes of phenyl groups, for example in 75 methamphetamine hydrochloride ${ }^{35}$, occur at terahertz frequencies. It is also well established that the unit cell contracts as the temperature decreases, similar to aggregation. Furthermore, strong temperature dependence is observed in the solution state for AIE molecules, which is expected to be due to a correlation 80 between molecular rotations and fluorescence ${ }^{12,36}$. Consequently, we expect that a temperature dependent THz-TDS study in the solid state of a simple AIE molecule (TPE) could provide a direct measurement to test the validity of the RIR hypothesis as the dominant fluorescence enhancement mechanism for these 85 molecules.

\section{Experimental Methods}

Tetraphenylethene (TPE) was prepared according to the literature method $^{37}$. Single crystals of TPE were obtained by evaporating solvent slowly from its dichloromethane/methanol $(1: 1 \mathrm{v} / \mathrm{v})$ 90 solution.

For the THz-TDS measurements, TPE was co-mixed with polyethylene (PE) powder and compressed into pellets approximately $3 \mathrm{~mm}$ thick containing $300 \mathrm{mg}$ of material with a TPE:PE ratio of 1:4. An additional $240 \mathrm{mg}$ pellet of pure PE was 95 used as a reference. Variable temperature experiments were performed under vacuum using a continuous flow liquid-nitrogen 
cooled cryostat (Janis ST-100, Janis Research) and temperature controller (Lakeshore 331, Lakeshore Cryotronics Inc). Terahertz spectra were obtained, with $5 \mathrm{~K}$ steps between 80 and $130 \mathrm{~K}$, and $10 \mathrm{~K}$ steps between 130 and $290 \mathrm{~K}$, according to methods ${ }_{5}$ previously reported ${ }^{38}$. The use of a silicon beam stop to block the reflected pump beam resulted in a small post peak pulse that was removed using linear modelling techniques ${ }^{39}$.

Single crystal X-ray intensity data were collected at $100 \mathrm{~K}$ and room temperature on a Bruker-Nonices Smart Apex CCD 10 diffractometer with graphite monochromated Mo-K $\alpha$ radiation. Processing of the intensity data was carried out using the SAINT and $\mathrm{SADABS}^{40}$ routines and the structure and refinement were conducted using the SHELXTL ${ }^{41}$ suite of X-ray programs (version 6.10). The $100 \mathrm{~K}$ structure has been previously 15 published e.g. CCDC no. $633293^{42}$ but both structures are provided as supplementary information.

Computational Density Functional Theory (DFT) calculations for solid state TPE at $110 \mathrm{~K}$ and $290 \mathrm{~K}$ were performed using the academic version of CASTEP ${ }^{43}$, using standard norm-conserving 20 pseudo-potentials and the PBE functional ${ }^{44}$. All calculations used the following parameters: k-point separation of $0.05 \AA^{-1}$, plane wave basis cutoff of $1200 \mathrm{eV}$, electronic convergence tolerance of $\Delta \mathrm{E}<1 \times 10^{-13} \mathrm{eV} /$ atom. In addition, we used the semi-empirical dispersion correction (d-DFT) scheme first formulated by ${ }_{25}$ Grimme $^{45,46}$ to account for the London-type dispersion forces that are expected to dominate the inter-molecular behaviour. Geometry optimizations were performed to the following tolerances: energy $<5 \times 10^{-8} \mathrm{eV} /$ atom, ionic forces $<3 \times 10^{-5} \mathrm{eV} / \AA$ and ionic displacement $<1 \times 10^{-5} \AA$. In order to incorporate the 30 dispersion correction in the phonon mode calculations, the finite displacement phonon calculation method was employed ${ }^{47}$, using a displacement amplitude of $1 \times 10^{-2} \AA$.

Photoluminescence (PL) measurements of TPE pellets were performed by exciting the same pellets as used for THz-TDS 35 measurements with an Omnichrome $325 \mathrm{~nm} \mathrm{He}-\mathrm{Cd}$ laser. The PL signal was measured by a SPEX 500 monochrometer and a photomultiplier tube (TE177RF003 Hamamatsu, Japan) at temperatures between 20 and $290 \mathrm{~K}$ (Expander DE202, APD Cryogenics, PA).

\section{${ }_{40}$ Results and Discussion}

\section{THz-TDS measurements}

Fig. 1(a) shows the evolution of the absorption coefficient ( $\alpha$ ) for TPE as a function of temperature over the measured frequency range $(0.1-2.2 \mathrm{THz})$. For clarity, example line plots taken at ${ }_{45} 280 \mathrm{~K}$ and $80 \mathrm{~K}$ (Fig. 1(b)) are also plotted, to highlight the main features of the high and low temperature spectra. To test whether isomerisation of the structure was a factor in the fluorescence ${ }^{48}$, terahertz spectra were obtained both with and without UV illumination. No difference (to within experimental error) was 50 observed between the illuminated and non-illuminated terahertz spectra at any of the temperatures measured (an example of this is shown in the supplementary information). At least three spectral features can be identified at both high and low temperatures. These are a shoulder at $1.0 \mathrm{THz}$ and peaks at 1.55 and $1.87 \mathrm{THz}$ 55 in the high temperature spectrum, and a peak at $1.2 \mathrm{THz}$, a shoulder at $1.73 \mathrm{THz}$, and a sharp peak at $1.94 \mathrm{THz}$ in the low temperature spectrum. In addition, a further small peak can be observed at $1.52 \mathrm{THz}$ in the low temperature spectrum. Integration of the areas under the absorption features shows that 60 the high temperature spectrum has a $38 \%$ higher absorption in this frequency region when compared to the low temperature spectrum. The change in composition and intensity of spectral features suggests a change in the structure of the supramolecular arrangement with temperature. To explore this possibility, we 65 calculated the vibrational modes for both the low and high temperature structures of TPE using the software package CASTEP, based upon crystal structures that were calculated from single crystal X-ray diffraction experiments.
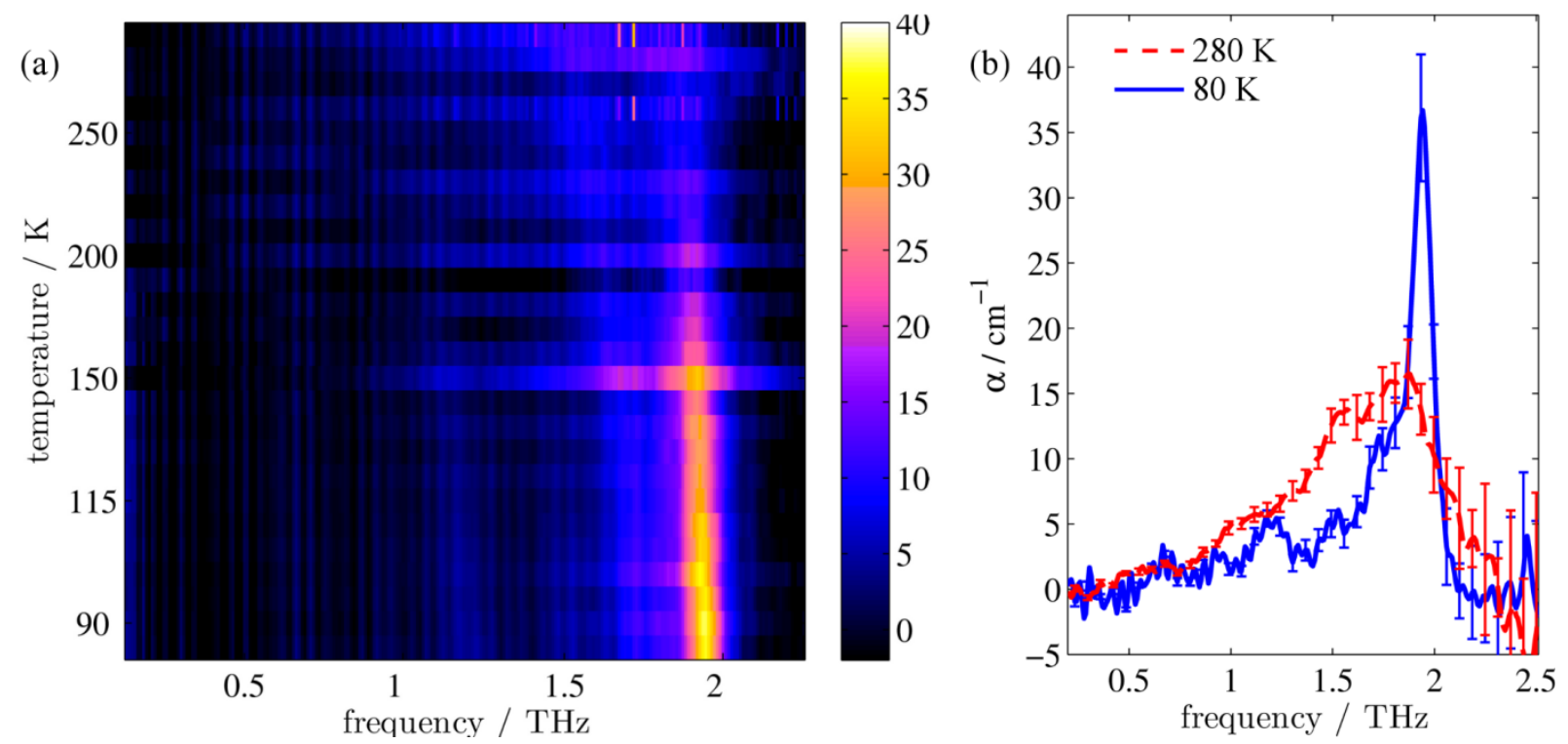

70 Fig. 1 (a) Imagemap of the change in absorption coefficient for TPE with temperature. (b) shows representative absorption spectra for TPE at the high (red dashed line) and low (blue solid line) temperatures. 

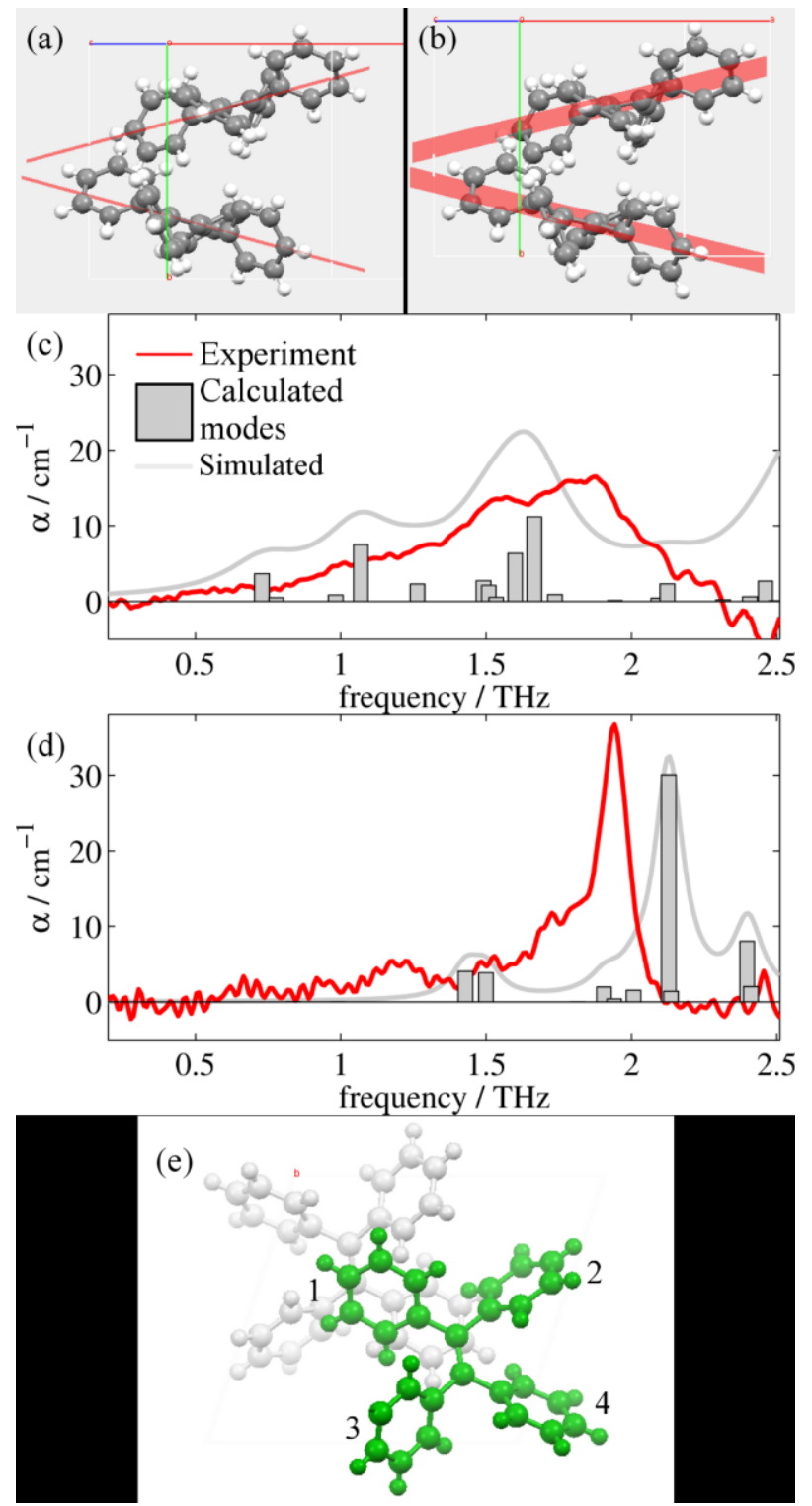

(e)
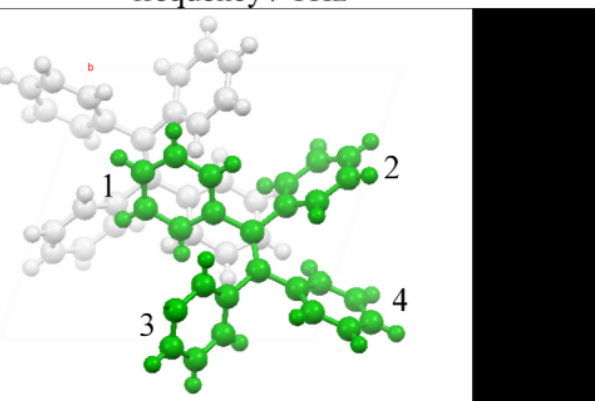

Fig. 2 Plane orientations for the (a) high temperature and (b) low temperature structures viewed along the $\mathrm{c}$ axis, with the $\mathrm{C}=\mathrm{C}-\mathrm{Ph} 1$ plane highlighted in red. Comparison of the predicted CASTEP vibrational

intensities from the (c) high temperature and (d) low temperature structures, respectively. The simulated spectra use Lorentzian lineshapes with linewidths of 5 and $2 \mathrm{~cm}^{-1}$ respectively. (e) Low temperature unit cell viewed along the crystallographic b-axis with the two molecules represented in different colours. The phenyl numbering is used to assign 10

\section{Calculation of the Vibrational Modes}

Analysis of the high and low temperature unit cells revealed small changes in the torsion angles of the four phenyl groups between the two structures (see Supplementary Table 1). These 15 changes ranged between $0.60^{\circ}$ and $4.00^{\circ}$ in magnitude, with the smallest change observed for the ph2 moieties. Furthermore, the orientation of the two molecules within the unit cell is observed to change. Fig. 2(a) and (b) shows the differences in the two unit cells by displaying planes generated by the $\mathrm{C}=\mathrm{C}-\mathrm{Ph} 1$ bonds.

20 d-DFT enabled geometry optimizations were performed for both the high and low temperature structures. Initially, the unit cell was not fixed, and the resulting crystal structure parameters found are detailed in Supplementary Table 2. The two experimental structures optimized to very similar final structures; 25 so we determined the suitability of the d-DFT derived structure by comparing the rms Cartesian displacement for the experimental and optimized structures ${ }^{49}$. The high temperature structure had an rms Cartesian displacement of $0.29 \AA$ ( $\max$ displacement was $0.58 \AA$ ) suggesting a temperature effect that 30 cannot be modelled by the geometry optimization ${ }^{49,50}$. In contrast, the low temperature structure only had an rms displacement of $0.11 \AA$, well within the typical displacement observed for these geometry optimizations ${ }^{49}$. Therefore, it was decided to fix the unit cell dimensions for the high temperature

35 structure and perform phonon calculations on the resulting optimised structure (note that the d-DFT corrections were still used for these calculations). Given the close agreement with the experimental structure, the non-unit-cell-fixed structure was used for the phonon calculations of the low temperature structure.

40 Fig. 2(c) and (d) show the mode frequencies and intensities predicted by CASTEP for the high and low temperature structures, respectively, as well as simulated spectra and the measured absorption spectra from our measurements. Supplementary Table 3 details the vibrational modes calculated 45 for each structure in this region along with descriptions of the significant modes, which are intramolecular rotations and vibrations of the phenyl (ph) groups. Whilst being superficially symmetric, the TPE molecule is actually asymmetric in the solid state, as each of the ph groups has different sets of nearest 50 neighbour interactions and consequently different equilibrium positions and angles. There are small offsets in the frequency of some of the modes which is common in these calculations ${ }^{52}$, and the relative intensities and positions of the vibrational modes agree more closely for the low temperature structure than the high 55 temperature structure. This is not surprising, given the limitations we have observed when optimizing and performing phonon calculations on the high temperature structure, as the current CASTEP implementation does not account for the temperature dependent structural evolution observed for TPE; 60 consequently the optimized high temperature structure is not as accurate as the low temperature version. However, the overall trend of the two structures is reproduced satisfactorily, with the shoulder and peaks being reproduced in both the high and low temperature simulations with approximately the correct relative 65 intensities. Closer analysis of the types of vibrational modes (Supplementary Table 3) suggest that whilst at high temperatures the modes are dominated by movements of ph1 and ph4 groups, at low temperatures the motions are dominated by the movements of the ph2 group (and to a smaller extent the ph1 70 group), in particular that of the large vibrational mode at $2.13 \mathrm{THz}$.

\section{TPE structure evolution during unit cell contraction}

The changes in relative intensities of the vibrational modes found using theoretical modelling are also seen in our THz-TDS 75 measurements. In these next sections we carefully analyse the $\mathrm{THz}$ measurement data and relate the spectral changes to the transitions occurring in the TPE molecule. From Fig. 1(a) we see that the terahertz data has interesting features between 1.45 and $2.05 \mathrm{THz}$ : as the temperature is increased, the low temperature 80 peak at $1.94 \mathrm{THz}$ loses intensity and shifts to $1.87 \mathrm{THz}$, and 
similarly the $1.73 \mathrm{THz}$ peak shifts to $1.55 \mathrm{THz}$. We hypothesize that the terahertz spectra of the high and low temperature structures can be broadly modelled using these two peaks, which themselves will be a superposition of multiple low/high 5 frequency modes predicted by the computational models in the previous section. As the peaks are relatively close in frequency (compared with their widths) their spectral lines will overlap and so we have fitted the data with two Lorentz oscillator functions (over the region 1.45-2.05 THz). Results of this fitting are shown 10 for four of the temperatures in Fig. 3(a)-(d) (the rest are given in the supplementary information).
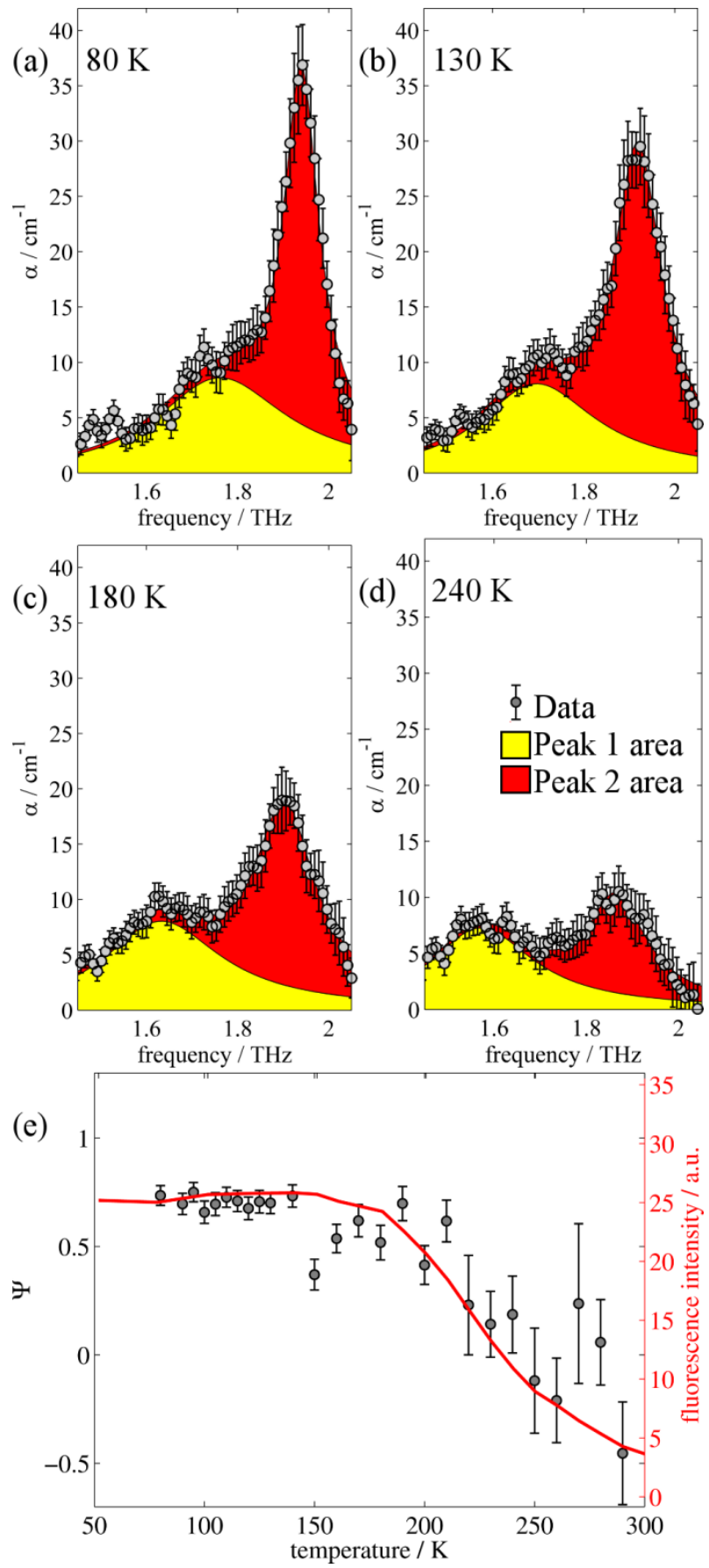

Fig. 3 (a) - (d) Two Lorentz function fits to the data for $80 \mathrm{~K}, 130 \mathrm{~K}$, $180 \mathrm{~K}$, and $240 \mathrm{~K}$ respectively between 1.45 and $2.05 \mathrm{THz}$. (e)
15 Comparison of PL measurements and the relative change in peak size as measured by THz-TDS as a function of temperature.

From the fits we extract the relative heights of the two spectral features (independently of each other), which we denote as $P_{1}$ and $P_{2}$. At low temperatures, the spectrum has a large $P_{2}$ 20 component and a relatively small $P_{1}$ component, whereas at high temperatures, the spectrum has $P_{1}$ and $P_{2}$ components that are more similar in intensity. Consequently, the ratio of these values is a way to estimate the relative strengths of the vibrational modes in these regions at a particular temperature. Equation 1 ${ }_{25}$ defines a strength ratio, $\Psi$, such that:

$$
\Psi=\frac{P_{2}-P_{1}}{P_{2}}
$$

$\Psi$ is plotted in Fig. 3(e) for measurements between 80 and $290 \mathrm{~K}$ along with the fluorescence intensity measured at the same temperatures. The observed similarity in the evolution of this 30 relative measure of the two features and that of the photoluminescence (PL) fluorescence intensity with temperature is marked. There are some outliers, noticeably at $150 \mathrm{~K}$ and also at 270 and $280 \mathrm{~K}$ but these can be attributed in part to baseline measurement issues (the former) and difficulties in accurately 35 fitting the function at higher temperatures due to the broadness and overlapping nature of the response. Despite this, there appears to be a compelling correlation between the relative peak intensity and therefore the change in vibrational modes, and the fluorescence of TPE due to the AIE phenomenon. The 40 observation that the fluorescence intensity does not further increase below $150 \mathrm{~K}$ is counter intuitive - as the lower temperature decreases separation and thus increased intensity would be expected. Why does the intensity not increase further? We address this question in the next section by examining $P_{2}$ in 45 more detail.

\section{Uneven conversion of vibrational modes?}

Closer inspection of the large low temperature peak (P2) at $1.94 \mathrm{THz}$ reveals a further shoulder at $1.88 \mathrm{THz}$ that increased in intensity with increasing temperature whilst the original main 50 peak decreased in intensity, until the $1.88 \mathrm{THz}$ feature became the dominant mode (at high temperatures). From our observations in the previous section, we deduce that this is due to the conversion of the dominant vibrational mode in this region from the low temperature to the high temperature version; furthermore the 55 vibrational analysis in Supplementary Table 3 suggested that the vibrations tentatively assigned to this feature are dominated by the ph4 moiety at high temperatures yet display mostly ph2 (and some ph4) character at low temperatures. We therefore performed similar analysis to that done previously, but this time fitted two ${ }_{60}$ Lorentz functions to the data between $1.82-2.05 \mathrm{THz}$ to observe how this peak changes with temperature. We chose to model this peak using two discrete functions as the high and low temperature structures predicted only a small number of vibrations closely spaced in frequency. Results of this fitting are shown for four of ${ }_{65}$ the temperatures in Fig. 4(a)-(d) (the rest are given in the supplementary information). 

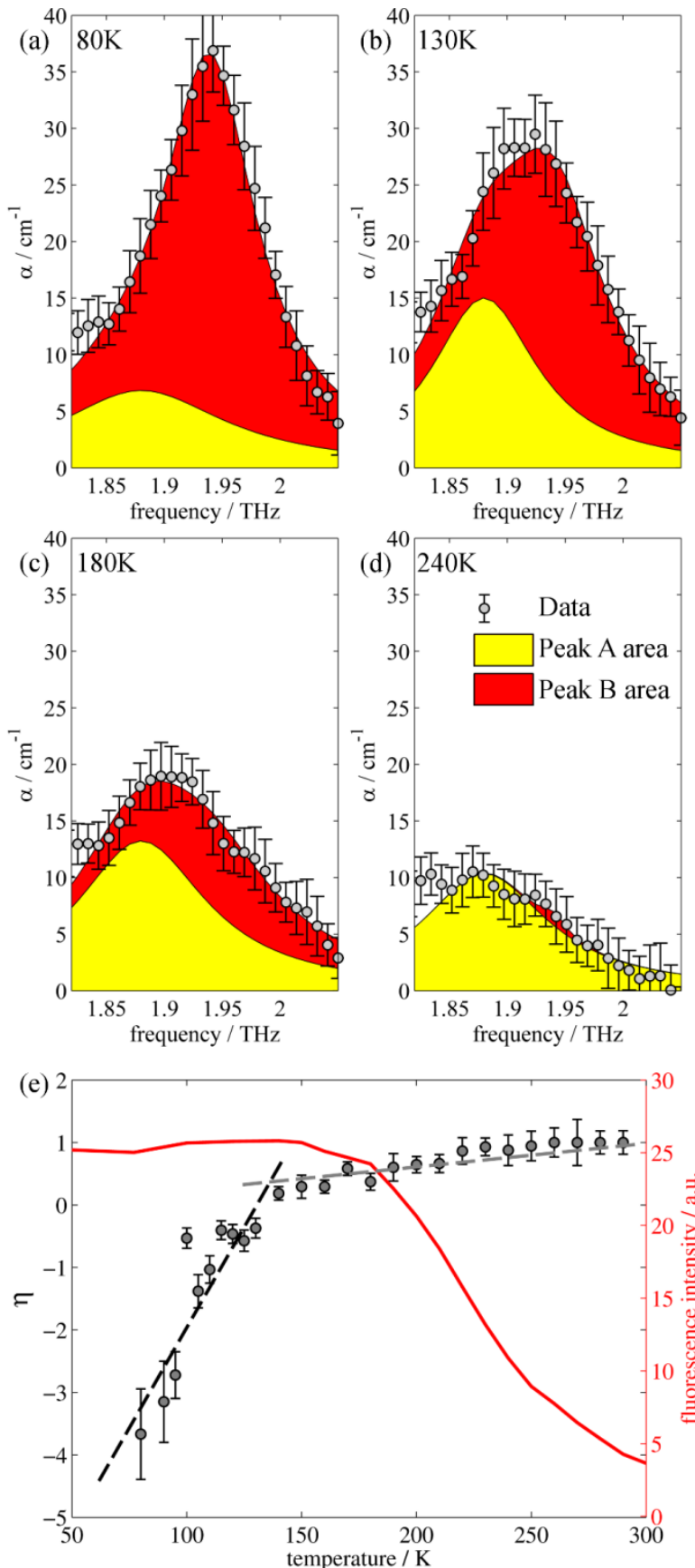

Fig. 4 (a) - (d) Two Lorentz function fits to the data for $80 \mathrm{~K}, 130 \mathrm{~K}$,

$180 \mathrm{~K}$, and $240 \mathrm{~K}$ respectively between 1.8 and $2.05 \mathrm{THz}$. (e) Relative change in peak size as measured by THz-TDS as a function of temperature. The PL data is plotted for comparison.

The use of two vibrational modes to fit this peak worked well. In particular it was able to accurately reproduce the asymmetric peak shape observed for the intermediate temperatures (for example at $130 \mathrm{~K}$ as shown in Fig. 4(b)). Following a similar 10 theory as in the previous section, we can use a measure of the ratio of the two peak heights to estimate the relative strengths of the high $\left(\mathrm{P}_{\mathrm{a}}\right)$ and low $\left(\mathrm{P}_{\mathrm{b}}\right)$ temperature modes:

$$
\eta=\frac{P_{a}-P_{b}}{P_{a}}
$$

$\eta$ is plotted in Fig. 4(e) and we can see that the evolution of this 15 ratio with temperature is very different to the evolution observed for the broader peaks (and the PL data). Namely that the high temperature mode increases relative to the low temperature mode from $80 \mathrm{~K}$, and by approximately $140 \mathrm{~K}$ the high temperature mode dominates this feature. In contrast to the results in Fig. 3(e) 20 for $P_{1}$ and $P_{2}$, above $140 \mathrm{~K}$ there is just a gradual decrease of the low temperature mode despite the rapid decrease in fluorescence intensity. This suggests that the evolution of the high frequency terahertz modes from the low to high temperature versions have a different effect on the fluorescence than that which was observed 25 in the previous section. Above $140 \mathrm{~K}$, where the fluorescence is observed to drop, the high frequency modes exhibit little change. Below $140 \mathrm{~K}$, where the modes are observed to transform from the high temperature ph4 dominated modes to the low temperature ph2 (and ph4) dominated modes, the fluorescence 30 exhibits little change.

The evidence that the high temperature and low temperature structures are different and not merely a unit cell expanded by different amounts has already been mentioned when the vibrational mode calculations were first introduced. Therefore to

35 understand the results from the terahertz measurements it is useful to return to this observation. Firstly, the unit cell contraction from high to low temperatures is asymmetric, from $9.8370,9.5017$ and $10.7087 \AA(a, b, c)$ for the high temperature structure and 9.7771, 9.1722 and $10.7780 \AA(a, b, c)$ for the low 40 temperature structure, implying that the majority of this compression would occur along the $b$ axis. However, the rms Cartesian displacement of the atoms in the unit cell is very different for the optimized high and low temperature unit cells. When the unit cell is allowed to relax, the resultant low 45 temperature structure is very close to the experimental result (a small rms displacement of $0.11 \AA$ ), whereas the high temperature structure alters a lot (a relatively large rms displacement of $0.29 \AA$ ). This is indicative of a subtle temperature dependent structural change. Yet the evolution of the terahertz dependent so vibrational modes suggests that this structural change is a gradual change, as evidenced by the evolution of particular vibrational features. Whilst a consideration of the overall spectrum reveals a structural evolution consistent with the PL changes, the evolution of a particular vibrational mode has a different temperature $5 s$ dependency.

The two temperature structures differ in the strength and distribution of the London dispersion forces within the unit cell. These intermolecular forces serve to dampen or restrict the intramolecular modes; consequently when the TPE crystal is ${ }_{60}$ cooled the force distribution restricts the intramolecular motions of the ph1 moiety (in particular); these are the dominant terahertz features at high temperatures. As a result, the energy losses from the intramolecular rotations of ph1 are quenched. Instead, further energy losses are dominated by rotational modes of the ph2 (and $\left.{ }_{65} \mathrm{ph} 4\right)$ moieties that are less strong than the ph1 dominated modes. It is the quenching of the ph1 dominated rotations that result in the observed increase in fluorescence, which is consistent with the RIR hypothesis. However, energy losses associated with the modes dominated by the ph2 (and ph4) group remain, a result that 70 appears to be due, in part, to the subtle structural change of the unit cell with temperature. Consequently, there is a point where, 
despite more contraction of the unit cell further suppressing the ph1 dominated modes, the ph2 (and ph4) dominated modes act to suppress any further fluorescence enhancement, due to their relative freedom, resulting in the plateau in fluorescence intensity 5 observed below $150 \mathrm{~K}$. Again, this observation from the terahertz and the crystal structures provides experimental evidence to support the RIR hypothesis, as we see that if the intramolecular modes are not restricted by intermolecular forces then the fluorescence does not increase.

10 What is particularly significant about both the terahertz spectral evolution observed herein and the computational calculations undertaken is that it is revealing subtle structural changes which are insufficient to be termed a "phase change". Despite this, these changes are enough to significantly alter the 15 fluorescent behaviour of the molecule. To our knowledge, previous terahertz spectral studies of this type have focused on following phase changes and dehydration kinetics ${ }^{53,25,54}$; this is the first demonstrating the sensitivity to subtle structural evolution that has clearly observable consequences to the material 20 behaviour.

\section{Conclusions}

This work represents the first direct evidence of a restriction of intramolecular modes being the dominant mechanism of emission enhancement in AIE molecules. These low frequency modes fall 25 into the terahertz frequency range and so we were able to follow the terahertz spectral evolution of this material with temperature, where we observed a change in the features present in the terahertz spectrum, in particular a $38 \%$ increase in the integrated terahertz spectral intensity at high temperature compared to low 30 temperature. The fact that no change was observed upon UV illumination suggested that isomerisation was not occurring, as this would change the TPE spectra. Using structures determined from single crystal X-ray studies at both room and low temperatures, we performed computational simulations of the 35 vibrations using CASTEP and found evidence of a subtle structural evolution with temperature (that hampered the high temperature phonon mode calculations) that satisfactorily predicted the observed change in the terahertz spectra. Furthermore, we observed that the relative heights of two spectral 40 regions appeared to change between low and high temperature, and when the ratio between these was compared with the observed fluorescence intensity we observed a correlation. Using terahertz spectroscopy and computational modelling we found that it is the restriction of intramolecular modes dominated by 45 phenyl group 1 causing the increase in fluorescence intensity, which we believe is the first time such a subtle structural evolution has been quantitatively measured using terahertz spectroscopy. Finally, study of the calculated vibration modes and crystal structures revealed that further enhancement of the ${ }_{50}$ AIE effect was hindered by the relative freedom of the phenyl 2 (and 4) group within the low temperature structure, which we hypothesised results from the intermolecular forces felt by the TPE molecule. There remains, however, a complex interplay between an optimum stacking arrangement to restrict 55 intramolecular rotation, and $\pi-\pi$ stacking interactions that would lead to other non-radiative energy loss processes ${ }^{5,55}$. Nevertheless, these results suggest that the restriction of intramolecular modes, brought about by the subtle temperaturedependent structural evolution, is the dominant enhancement ${ }_{60}$ mechanism for the fluorescence behaviour of TPE in the solid state.

\section{Acknowledgements}

The authors would like to thank Lixia Zhang and Jiannong Wang for their help with the fluorescence measurements, Herman ${ }_{65} \mathrm{H}$. Y. Sung and Ian D. Williams for the single crystal structure data, and $\mathrm{X}$. W. Wan and F. Thomas for preliminary discussions. In addition the authors would like to acknowledge the useful comments of the reviewers, and D. Tomerini and A. D. Burnett for useful discussions regarding the computational aspects. This 70 work was partially supported by the RPC grant of HKUST (RPC11SC09), the Research Grants Council of Hong Kong (616611 HKUST2/CRF/10 and N_HKUST620/11), the University Grants Committee of HK (AoE/P-03/08), HKUST project of merit (FSGRF13EG33) and CUHK Direct Grant 75 (4055001). N.Y.T. would like to acknowledge the Agency for Science, Technology and Research for PhD funding.

\section{Notes and references}

${ }^{a}$ Department of Electronic Engineering, The Chinese University of Hong Kong, Shatin, N.T., Hong Kong. Fax: (852) 2603 5558; Tel: (852) 3943 808260; E-mail:emma@ee.cuhk.edu.hk

${ }^{b}$ Department of Chemical Engineering and Biotechnology, University of Cambridge, Pembroke Street, Cambridge CB2 3RA, UK. Fax: (+44) 1223 334796; Tel: (+44) 1223 334783; E-mail: jaz22@cam.ac.uk

${ }^{c}$ Department of Chemistry, Institute of Molecular Functional Materials 85 and Division of Biomedical Engineering, The Hong Kong University of Science and Technology, Clearwater Bay, Kowloon, Hong Kong. Fax: (+852) 2358 1594; Tel: (+852) 2358 7375; E-mail:tangbenz@ust.hk

$\dagger$ Electronic Supplementary Information (ESI) available: Evidence of the insensitivity of the terahertz measurements to UV illumination, 90 crystallographic information files (CIF) for the high and low temperature TPE strutures, tables indicating the torsional angles, crystal structure paramaters and the full vibrational assignments for the low and high temperature TPE structures,, and the full fitting results for the Lorentz fitting functions in Figs. 3 and 4 are presented as supplementary 95 information. See DOI: 10.1039/b000000x/

\$ Footnotes should appear here. These might include comments relevant to but not central to the matter under discussion, limited experimental and spectral data, and crystallographic data.

1. M. Wang, D. Zhang, G. Zhang, Y. Tang, S. Wang, and D. Zhu, Anal. Chem., 2008, 80, 6443-6448.

2. T. Sanji, K. Shiraishi, M. Nakamura, and M. Tanaka, Chem. Asian J., 2010, 5, 817-24.

3. S. Kim, H. E. Pudavar, A. Bonoiu, and P. N. Prasad, Adv. Mater., 2007, 19, 3791-3795.

105 4. C.-K. Lim, S. Kim, I. C. Kwon, C.-H. Ahn, and S. Y. Park, Chem. Mater., 2009, 21, 5819-5825.

5. J. B. Birks, Photophysics of aromatic molecules, Wiley, London, 1970.

6. S. M. Borisov and O. S. Wolfbeis, Chem. Rev., 2008, 108, 423-61.

1107 . U. Lemmer, S. Heun, R. F. Mahrt, U. Scherf, M. Hopmeier, U. Siegner, E. O. Go"bel, K. Mu"llen, and H. Ba"ssler, Chem. Phys. Lett., 1995, 240, 373-378.

8. J. Wang, Y. Zhao, C. Dou, H. Sun, P. Xu, K. Ye, J. Zhang, S. Jiang, F. Li, and Y. Wang, J. Phys. Chem. B, 2007, 111, 5082-9.

115 9. L. Chen, S. Xu, D. McBranch, and D. Whitten, J. Am. Chem. Soc., 2000, 122, 9302-9303.

10. S. Hecht and J. M. J. Frechet, Angew. Chem. Int. Ed., 2001, 40, 7491. 
11. J. Luo, Z. Xie, J. W. Y. Lam, L. Cheng, H. Chen, C. Qiu, H. S. Kwok, X. Zhan, Y. Liu, D. Zhu, and B. Z. Tang, Chem. Commun., 2001, 381, 1740-1741.

12. J. Chen, C. C. W. Law, J. W. Y. Lam, Y. Dong, S. M. F. Lo, I. D. Williams, D. Zhu, and B. Z. Tang, Chem. Mater., 2003, 15, 15351546.

13. Q. Peng, Y. Yi, Z. Shuai, and J. Shao, J. Am. Chem. Soc., 2007, 129, 9333-9.

14. S. Yin, Q. Peng, Z. Shuai, W. Fang, Y.-H. Wang, and Y. Luo, Phys. Rev. B, 2006, 73, 205409.

15. Z. Li, Y. Dong, B. Mi, Y. Tang, M. Häussler, H. Tong, Y. Dong, J. W. Y. Lam, Y. Ren, H. H. Y. Sung, K. S. Wong, P. Gao, I. D. Williams, H. S. Kwok, and B. Z. Tang, J. Phys. Chem. B, 2005, 109, 10061-6.

15 16. K. Kokado and Y. Chujo, J. Org. Chem., 2011, 76, 316-319.

17. X. Fan, J. Sun, F. Wang, Z. Chu, P. Wang, Y. Dong, R. Hu, B. Z. Tang, and D. Zou, Chem. Commun., 2008, 381, 2989-2991.

18. N. B. Shustova, B. D. McCarthy, and M. Dincă, J. Am. Chem. Soc., 2011, 133, 20126-9.

20 19. Y. Xu, L. Chen, Z. Guo, A. Nagai, and D. Jiang, J. Am. Chem. Soc., 2011, 133, 17622-17625.

20. Q. Wu, C. Deng, Q. Peng, Y. Niu, and Z. Shuai, J. Comput. Chem., 2012, 33, 1862-1869.

21. M. Nakamura, T. Sanji, and M. Tanaka, Chem. Eur. J., 2011, 17, 5344-5349.

22. N.-N. Liu, S. Song, D.-M. Li, and Y.-S. Zheng, Chem. Commun., 2012, 48, 4908-4910.

23. Z. Liu, W. Xue, Z. Cai, G. Zhang, and D. Zhang, J. Mater. Chem., 2011, 21, 14487.

30 24. J. A. Zeitler, P. F. Taday, D. A. Newnham, M. Pepper, K. C. Gordon, and T. Rades, J. Pharm. Pharmacol., 2007, 59, 209-23.

25. J. A. Zeitler, P. F. Taday, K. C. Gordon, M. Pepper, and T. Rades, Chem. Phys. Chem., 2007, 8, 1924-1927.

26. K. L. Nguyen, T. Friscić, G. M. Day, L. F. Gladden, and W. Jones, 35 Nat. Mater., 2007, 6, 206-9.

27. E. P. J. Parrott, J. A. Zeitler, L. F. Gladden, S. N. Taraskin, and S. R. Elliott, J. Non-Cryst. Solids, 2009, 355, 1824-1827.

28. E. P. J. Parrott, J. A. Zeitler, G. Simon, B. Hehlen, L. F. Gladden, S. N. Taraskin, and S. R. Elliott, Phys. Rev. B, 2010, 82, 140203.

40 29. C. Kang, I. H. Maeng, S. J. Oh, J.-H. Son, T.-I. Jeon, K. H. An, S. C. Lim, and Y. H. Lee, Appl. Phys. Lett., 2005, 87, 041908.

30. C. Kang, I. H. Maeng, S. J. Oh, S. C. Lim, K. H. An, Y. H. Lee, and J.-H. Son, Phys. Rev. B, 2007, 75, 1-5.

31. E. P. J. Parrott, J. A. Zeitler, J. McGregor, S.-P. Oei, H. E. Unalan,

45 W. I. Milne, J.-P. Tessonnier, D. S. Su, R. Schlögl, and L. F. Gladden, Adv. Mater., 2009, 21, 3953-3957.

32. E. P. J. Parrott, J. A. Zeitler, J. McGregor, S.-P. Oei, H. E. Unalan, S.-C. Tan, W. I. Milne, J.-P. Tessonnier, R. Schlögl, and L. F. Gladden, J. Phys. Chem. C, 2009, 113, 10554-10559.

50 33. A. G. Markelz, S. Whitmire, J. R. Hillebrecht, and R. Birge, Phys. Med. Biol, 2002, 47, 3797-3805.

34. Y. Sun, Y.-T. Zhang, and E. Pickwell-Macpherson, Biophys. J., 2011, 100, 225-231.

35. P. M. Hakey, D. G. Allis, W. Ouellette, and T. M. Korter, J. Phys. Chem. A, 2009, 113, 5119-27.

36. R. Hu, E. Lager, A. Aguilar-Aguilar, J. Liu, J. W. Y. Lam, H. H. Y. Sung, I. D. Williams, Y. Zhong, K. S. Wong, E. Peña-Cabrera, and B. Z. Tang, J. Phys. Chem. C, 2009, 113, 15845-15853.

37. Y. Dong, J. W. Y. Lam, A. Qin, J. Liu, Z. Li, B. Z. Tang, J. Sun, and 60 H. S. Kwok, Appl. Phys. Lett., 2007, 91, 011111.

38. R. Li, J. A. Zeitler, D. Tomerini, E. P. J. Parrott, L. F. Gladden, and G. M. Day, Phys. Chem. Chem. Phys., 2010, 12, 5329-5340.

39. O. Hirsch, P. Alexander, and L. Gladden, Microelectron. J., 2008, 39, 841-848.

65 40. G. M. Sheldrick, 1996.

41. G. M. Sheldrick, Acta Crystallogr. A, 2008, 64, 112-22.

42. G. Hua, Y. Li, A. M. Z. Slawin, and J. D. Woollins, Dalt. T., 2007, $1477-1480$.

43. S. J. Clark, M. D. Segall, C. J. Pickard, P. J. Hasnip, M. I. J. Probert,

70 K. Refson, and M. C. Payne, Z. Krist., 2005, 220, 567-570.
44. J. P. Perdew, K. Burke, and M. Ernzerhof, Phys. Rev. Lett., 1996, 77, 3865-3868.

45. S. Grimme, J. Comput. Chem., 2006, 27, 1787.

46. E. R. McNellis, J. Meyer, and K. Reuter, Phys. Rev. B, 2009, 80, 205414.

47. G. J. Ackland, M. C. Warren, and S. J. Clark, J. Phys. Condens. Mat., 1997, 9, 7861-7872.

48. N.-W. Tseng, J. Liu, J. C. Y. Ng, J. W. Y. Lam, H. H. Y. Sung, I. D. Williams, and B. Z. Tang, Chem. Sci., 2012, 3, 493-497.

80 49. J. van de Streek and M. a Neumann, Acta Crystallogr. B, 2010, 66, 544-58.

50. A. D. Bond, K. a. Solanko, J. van de Streek, and M. a. Neumann, CrystEngComm, 2011, 13, 1768.

51. D. G. Allis, D. A. Prokhorova, and T. M. Korter, J. Phys. Chem. A, 2006, 110, 1951-9.

52. M. D. King, W. D. Buchanan, and T. M. Korter, Phys. Chem. Chem. Phys., 2011, 13, 4250-9.

53. H. Liu and X.-C. Zhang, Chem. Phys. Lett., 2006, 429, 229-233.

54. M. Franz, B. M. Fischer, and M. Walther, J. Mol. Struct., 2011, 1006, 34-40.

55. B. Valeur and M. N. Berberan-Santos, Molecular Fluorescence: Principles and Applications, Wiley-VCH, Weinheim, 2nd Ed., 2012. 